\title{
Short Wave Ultraviolet Light (UV-C) Effectiveness in the Inactivation of Bacterial Spores Inoculated in Turbid Suspensions and in Cloudy Apple Juice
}

\author{
Jezer N. Sauceda-Gálvez ${ }^{1}\left(\mathbb{D}\right.$, María Martinez-Garcia ${ }^{1}$, Ma Manuela Hernández-Herrero ${ }^{1}$ (D), Ramón Gervilla ${ }^{2}$ (D) \\ and Artur X. Roig-Sagués ${ }^{1, *(\mathbb{D})}$ \\ 1 Departament de Ciència Animal i dels Aliments, Facultat de Veterinària, Centre d'Innovació, Recerca i \\ Transfèrencia en Tecnologia dels Aliments (CIRTTA), XaRTA, TECNIO-CERTA, MALTA-Consolider Team, \\ Universitat Autònoma de Barcelona, 08193 Bellaterra, Spain; jezernoe.sauceda@e-campus.uab.cat (J.N.S.-G.); \\ martinez_maria91@yahoo.com (M.M.-G.); manuela.hernandez@uab.cat (M.M.H.-H.) \\ 2 SPTA-Servei Planta Tecnologia Aliments, Universitat Autònoma de Barcelona, c/de l'Hospital S/N, \\ 08193 Bellaterra (Barcelona), Spain; ramon.gervilla@uab.cat \\ * Correspondence: arturxavier.roig@uab.cat
}

check for updates

Citation: Sauceda-Gálvez, J.N.; Martinez-Garcia, M.

Hernández-Herrero, M.M.; Gervilla,

R.; Roig-Sagués, A.X. Short Wave

Ultraviolet Light (UV-C) Effectiveness in the Inactivation of Bacterial Spores Inoculated in Turbid Suspensions and in Cloudy Apple Juice. Beverages 2021, 7, 11. https://doi.org/10.3390/ beverages7010011

Academic Editor: Antonio Cilla

Received: 16 December 2020

Accepted: 7 February 2021

Published: 16 February 2021

Publisher's Note: MDPI stays neutra with regard to jurisdictional claims in published maps and institutional affiliations.

Copyright: (c) 2021 by the authors. Licensee MDPI, Basel, Switzerland. This article is an open access article distributed under the terms and conditions of the Creative Commons Attribution (CC BY) license (https:/ / creativecommons.org/licenses/by/ $4.0 /)$.

\begin{abstract}
Liquid foods might present interferences in their optical properties that can reduce the effectiveness of short-wave ultraviolet radiation (UV-C) treatments used for sterilization purposes. The effect of turbidity as UV-C interference factor against the inactivation of bacterial spores was analysed by using phosphate-buffered saline solutions (PBS) of different turbidity values (2000, 2500, and 3000 NTU) which were adjusted with the addition of apple fibre. These suspensions were inoculated with spores of Bacillus subtilis and Alicyclobacillus acidoterrestris. While higher UV-C doses increased the inactivation rates of spores, these were reduced when turbidity values increased; a dose of $28.7 \mathrm{~J} / \mathrm{mL}$ allowed inactivation rates of B. subtilis spores of $3.96 \mathrm{Log}$ in a 2000-NTU suspension compared with 2.81 Log achieved in the 3000-NTU one. Spores of B. subtilis were more UV-C-resistant than $A$. acidoterrestris. Cloudy apple juice inoculated with $A$. acidoterrestris spores was processed by UV-C at different doses in a single pass and with recirculation of the matrix through the reactor. Inactivation increased significantly with recirculation, surpassing $5 \mathrm{Log}$ after $125 \mathrm{~J} / \mathrm{mL}$ compared with 0.13 Log inactivation after a single-pass treatment at the same UV-C dose. UV-C treatments with recirculation affected the optical properties (absorption coefficient at $254 \mathrm{~nm}$ and turbidity) of juice and increased browning as UV-C doses became higher.
\end{abstract}

Keywords: short-wave ultraviolet radiation; sterilization; apple juice; turbidity; Alicyclobacillus acidoterrestris

\section{Introduction}

In recent years short-wave ultraviolet light (UV-C) has been tested as an alternative to thermal treatments in food industry, not only for the microbiological decontamination of surfaces, but also for the reduction of the microbial load in liquid foods [1]. Many of the currently sold UV-C machines for the pasteurisation of these products assure to meet the FDA requirements for the elimination of $E$. coli, but among the microorganisms that are potentially present in these foods, bacterial spores are the ones which oppose the highest resistance to thermal treatments commonly used for pasteurisation processes and to pressure-based technologies such as high-hydrostatic pressure and ultra-high-pressure homogenisation [2-4]. Once these spores contaminate foods and survive pasteurisation treatments, they germinate and grow even at low $\mathrm{pH}$ spoiling the final product meant to be shelf-stable [5]. That has been described in apple juice batches contaminated with spores of Alicyclobacillus acidoterrestris [6]. This bacterium might not produce any visible signs of deterioration, like gas or higher turbidity before reaching an excessive cell concentration, but although it is harmless to humans and represents no health hazard it spoils the product 
with the production of odd flavours and odours because of the synthesis of guaiacol making it not suitable for consumption. For that reason, it is considered a target microorganism for novel technologies for fruit juice processing $[7,8]$.

UV-C, which usually is emitted by lamps at a wavelength of $253.7 \mathrm{~nm}$ [9], has been proved to successfully inactivate microorganisms in their vegetative or spore state by penetrating through their membranes and being absorbed by their DNA which has a maximum absorption peak in the wavelength range between 254 and $264 \mathrm{~nm}$, forming pyrimidine dimers which blocks DNA transcription and replication processes leading to cell death [10]. Experiments performed in phosphate-buffered saline solutions (PBS), which opposes no interferences against UV-C $(254 \mathrm{~nm})$, demonstrate that a wide range of bacterial and fungal spores are susceptible to be inactivated by this technology $[3,11]$. Moreover, UV-C technology has been estimated to be cheaper than thermal pasteurisation, not only at the initial investment, but also at the energy required for processing foods that could approximately represent a 39\% of the total energy spent in thermally pasteurised fruit drinks like pineapple juice [12]. The main problem that UV-C has to face when treating foods is the interferences that are naturally present in them which will not allow light to penetrate through the matrix and reach microorganisms to inactivate them. The most important interferences found in foods are the absorption coefficient at the same wavelength as UV-C because it would not let it reach deep into the matrix, and the other one is the turbidity due to suspended particles which act as a shield to microorganisms and block the pass of UV-C light. To address these problems, many continuous-flow UV-C reactors have been designed to date. One of these approaches are the thin-film reactors that shorten the distance in which UV-C has to travel through, increasing the probability to reach as many cells as possible in order to inactivate them [13]. The purpose of this work was to evaluate the effectiveness of a concentric-ring thin-film UV-C reactor in inactivating bacterial spores inoculated in suspensions of different turbidity values as the main interference in order to know the degree in which those values can oppose to the effectiveness of UV-C to inactivate bacterial spores. For that purpose spores of Bacillus subtilis and of $A$. acidoterrestris were chosen because the first one has been commonly used as a model microorganism for the study of the characteristics of endospores [14], and the second one for being a target microorganism in the fruit juice industry due to their resistance to many types of stresses and for growing at low $\mathrm{pH}$ values [7]. The inactivation of $A$. acidoterrestris spores in cloudy apple juice with ascorbic acid, a real food matrix of high turbidity and absorption coefficient at $254 \mathrm{~nm}$, was studied by applying UV-C in a single pass or in recirculation. The inactivation kinetics of $A$. acidoterrestris following the later approach was also elucidated.

\section{Materials and Methods}

\subsection{Preparation of the Spore Suspensions}

Bacillus subtilis CECT 4002 and Alicyclobacillus acidoterrestris CECT 7094 were supplied from the Spanish Type Culture Collection (CECT, University of Valencia, Valencia, Spain). $B$. subtilis spores were obtained with a modified procedure based on the UNE EN ISO 13704:2002 as explained by [15]. The lyophile was rehydrated in glucose-tryptone broth consisting in $2.5 \mathrm{~g}$ of yeast extract (Oxoid, Basingstoke, UK), $5 \mathrm{~g}$ of tryptone (Oxoid) and $1 \mathrm{~g}$ of glucose (Sigma-Aldrich, St. Louis, MO, USA) per litre of distilled water, its $\mathrm{pH}$ was adjusted to 7.2 . It was incubated at $30^{\circ} \mathrm{C}$ for $24 \mathrm{~h}, 2 \mathrm{~mL}$ of this stock culture was transferred into Roux bottles containing yeast extract agar (MYA) which consisted in 10 $\mathrm{g}$ of meat extract (Oxoid), $2 \mathrm{~g}$ of yeast extract (Oxoid), $15 \mathrm{~g}$ of agar (Oxoid), and $0.04 \mathrm{~g}$ of MnSO4.H2O (Merck, Darmstadt, Germany) per litre of distilled water. These bottles were incubated at $30{ }^{\circ} \mathrm{C}$ for a maximum of 30 days to induce sporulation. Spores were collected by pouring $20 \mathrm{~mL}$ of sterile distilled water into each bottle and the surface was scraped with a Digralsky stick, the suspension was collected inside 50-mL Falcon tubes and centrifuged at 10,000 $\times g$ for 20 min at $4{ }^{\circ} \mathrm{C}$ using a Sigma $4 \mathrm{~K} 15$ centrifuge (Sigma Laborzentrifugen $\mathrm{GmbH}$, Osterode am Harz, Germany), the supernatant was discarded and the resulting 
pellet was washed with $15 \mathrm{~mL}$ of sterile water and centrifuged with the same settings, this procedure was done four times to be finally resuspended in $30 \mathrm{~mL}$ of sterile distilled water. The suspensions were heated at $75^{\circ} \mathrm{C}$ for $10 \mathrm{~min}$ in order to inactivate vegetative cells.

A. acidoterrestris spores were recovered in $\mathrm{K}$ broth as explained by [7] by incubating them at $43{ }^{\circ} \mathrm{C}$ for $48 \mathrm{~h}$. The stock culture was refrigerated at $4{ }^{\circ} \mathrm{C}$. Sporulation was induced by transferring $1 \mathrm{~mL}$ of the stock culture to $250-\mathrm{mL}$ Roux bottles containing $50 \mathrm{~mL}$ of potato dextrose agar (PDA) (Oxoid, Basingstoke, UK), and incubated at $43{ }^{\circ} \mathrm{C}$ from 7 to 15 days until reaching $80 \%$ sporulation or higher. Spores were collected by pouring $30 \mathrm{~mL}$ of sterile distilled water into each bottle and the surface was scraped with a Digralsky stick, the suspension was collected inside 50-mL Falcon tubes and centrifuged at 10,000 $\times g$ for 20 min at $4{ }^{\circ} \mathrm{C}$, the supernatant was discarded and the resulting pellet was washed with $30 \mathrm{~mL}$ of sterile water and centrifuged with the same settings, this procedure was done four times to be finally resuspended in $40 \mathrm{~mL}$ of sterile distilled water and heated at $80^{\circ} \mathrm{C}$ for $20 \mathrm{~min}$ in order to inactivate vegetative cells.

Spore preparations were placed in an ice bath after heat treatment for $10 \mathrm{~min}$ and stored at $4{ }^{\circ} \mathrm{C}$ until used.

\subsection{Matrices Preparation}

Phosphate-buffered saline (PBS) (Panreac Química S. L. U., Castellar del Vallès, Spain) with a $\mathrm{pH}$ of 7.4 was added with apple fibre (PBS-fibre) (The Hut Group, Manchester, UK) until reaching the turbidity values of 2000, 2500 and 3000 NTU (Nephelometric Turbidity Unit). Apple juice (Golden delicious var.) was obtained from a local producer. The cold-pressed apple juice was added with $300 \mathrm{ppm}$ of ascorbic acid at the moment of its extraction according to the producer. The matrices were inoculated with B. subtilis spores (final concentration $\sim 5.5 \log \mathrm{CFU} / \mathrm{mL}$ ) and A. acidoterrestris spores (final concentration $\sim 4.5$ to $5.3 \mathrm{Log} \mathrm{CFU} / \mathrm{mL}$ ) right before UV-C treatments.

\subsection{UV-C Treatments}

These treatments were done in a thin-film concentric-ring type UV-C reactor [16] (UV-Therm, Ypsicon S.L., Barcelona, Spain) of 70-mL capacity, with dimensions of 76.5-cm long and 1-mm width. This reactor has a feeding tank connected to a UV-C lamp (55 W) (LAB81055 NNI 150/76 XL, UV-Consulting Peschl España, Geldo, Spain) coated with quartz, and the lamp temperature is controlled with an air-cooling system. The lamp's irradiance was measured by actinometry with a potassium iodide/iodate solution as explained by the method of [17]. PBS suspensions were irradiated in a single pass through the reactor with the doses of $14.3,21.5$, and $28.7 \mathrm{~J} / \mathrm{mL}$. Apple juice was irradiated with the doses of $25,50,75,100$, and $125 \mathrm{~J} / \mathrm{mL}$ for microbiological analysis in a single pass and in recirculation through the reactor at a pumping speed of $0.708 \mathrm{~m} / \mathrm{s}$. In the same way, samples were irradiated at the doses of 50,100,150, and $200 \mathrm{~J} / \mathrm{mL}$ for physicochemical analysis. The time needed for an irradiation and the number of entrances was calculated as explained by [15] and that information can be found in Table 1. Samples destinated for microbiological analysis were refrigerated at $4{ }^{\circ} \mathrm{C}$, and samples destinated for physicochemical analysis were deposited in 50-mL Falcon tubes and stored at $-80^{\circ} \mathrm{C}$. 
Table 1. Calculated treatment time in minutes and seconds, and the number of entrances per litre of apple juice through the UV-C reactor at the different doses. The pumping speed for all doses was $0.708 \mathrm{~m} / \mathrm{s}$.

\begin{tabular}{ccc}
\hline UV-C Dose $(\mathbf{J} / \mathbf{m L})$ & $\begin{array}{c}\text { Treatment Time Per L of } \\
\text { Juice }\end{array}$ & Number of Entrances \\
\hline 25 & $14^{\prime} 52^{\prime \prime}$ & 60 \\
50 & $29^{\prime} 43^{\prime \prime}$ & 120 \\
75 & $44^{\prime} 35^{\prime \prime}$ & 180 \\
100 & $59^{\prime} 26^{\prime \prime}$ & 240 \\
125 & $74^{\prime} 18^{\prime \prime}$ & 300 \\
150 & $89^{\prime} 10^{\prime \prime}$ & 360 \\
200 & $118^{\prime} 53^{\prime \prime}$ & 480 \\
\hline
\end{tabular}

Reynolds Number Calculation in UV-C Treatments

Reynolds number $(R e)$ was calculated with Equation (1):

$$
\operatorname{Re}=\rho V D / \mu .
$$

where $V$ is fluid's velocity in $\mathrm{m} / \mathrm{s}, D$ is the equivalent diameter of $0.001632 \mathrm{~m}$ [18]. Dynamic viscosity $(\mu)$ was measured at $20{ }^{\circ} \mathrm{C}$ with a Haake RheoStress1 rheometer (Thermo Fisher Scientific, Inc., Karlsruhe, Germany) with a value for apple juice of $4.13 \pm 0.07 \times 10^{-3} \mathrm{~Pa}$. s. Density $(\rho)$ at $20^{\circ} \mathrm{C}$ was obtained with a density hydrometer (HYDR-100-001) (Labbox, Vilassar de Dalt, Spain) being of $1.052 \mathrm{~g} / \mathrm{mL}\left(1052 \mathrm{Kg} / \mathrm{m}^{3}\right)$ in apple juice.

\subsection{Microbiological Analysis}

Serial dilutions of the samples were done in sterile peptone water (Oxoid) and plated on petri dishes. Samples with B. subtilis were plated on tryptone-soya agar (Oxoid) using a double layer in order to prevent excessive colony growth. Plates were incubated at $30{ }^{\circ} \mathrm{C}$ for 2 days. A. acidoterrestris samples were plated on SK agar [19] and they were incubated at $43{ }^{\circ} \mathrm{C}$ for 3 days. Spore inactivation was calculated as the difference between the logarithmic counts of the control and the treated sample $\left(\log _{10} \mathrm{~N}_{0}-\log _{10} \mathrm{~N}\right)$.

\subsection{Physicochemical Analysis}

\subsubsection{Optical Properties}

Turbidity was measured with a turbidimeter Hach 2100Q (Hach Lange Spain, l'Hospitalet de Llobregat, Spain). Absorption coefficient at 254 nanometres wavelength $\left(\alpha_{254}\right)$ was measured by using a spectrophotometer NanoPhotometer Pearl (IMPLEN GmbH, München, Germany) and a quartz cuvette with a $1 \mathrm{~cm}$ pathlength. Samples were read at $20^{\circ} \mathrm{C}$.

\subsubsection{Browning Index}

It was determined following the method explained by [20]. Samples were filtered with syringe filters of $0.45 \mu \mathrm{m}$ pore diameter (Labbox) and mixed with ethanol in equal proportions. The absorbance of samples was read at $420 \mathrm{~nm}\left(A_{420}\right)$ with a spectrophotometer and results were expressed as $A_{420}$.

\subsection{Statistical Analysis}

Statistics and analysis of variance (ANOVA) were done by using the linear models (GLM) via software (Statistica ver 7, StatSoft, Inc, Tulsa, OK, USA). Tukey test was used for making comparisons between samples means. Differences were considered as significative when $p<0.05$.

\subsection{Inactivation Kinetics}

Survival data of $A$. acidoterrestris spores in apple juice was adjusted to a non-linear model by using GInaFiT software (version 1.7, MeBioS, Leuven, Belgium) add-in for 
Microsoft ${ }^{\circledR}$ Excel. The most suitable model was chosen according to the root mean square error and the $\mathrm{R}^{2}$ coefficient. The inactivation model that better adjusted to that data was Geeraerd model with shoulder and tail [21] which is represented in Equation (2):

$\left.\left.\log _{10}(N)=\log _{10}\left[\left(10^{\log _{10}\left(N_{0}\right)}-10^{\log _{10}\left(N_{\text {res }}\right)}\right) * e^{\left(-k_{\max } * t\right)} * e^{\left(k_{\max } * S l\right)}\right)\right] /\left[1+\left(e^{\left(k_{\max } * S l\right)}-1\right) * e^{\left(-k_{\max } * t\right)}\right)\right]+10^{\log _{10}\left(N_{\text {res }}\right)}$

where $N_{0}$ is the starting concentration of spores, $N_{\text {res }}$ is the number of survival subpopulations of resistant bacteria, $t$ means the time (in this adaptation it would be UV-C dose with recirculation), $k_{\max }$ is the inactivation rate of $0.15 \pm 0.01$ [1/time unit], and $S l$ [time unit] is the shoulder length of $15.64 \pm 3.64$.

\section{Results}

\subsection{UV-C Effectiveness in the Different Turbidity Suspensions}

As it can be observed in Figure 1, the inactivation of B. subtilis spores was inversely proportional to the turbidity value of the PBS-fibre suspensions, and within the same turbidity value, the inactivation of spores increased with higher UV-C doses. The highest inactivation $(3.96 \mathrm{Log})$ was obtained in the suspension of the lowest assayed turbidity (2000 NTU) and processed with the highest assayed UV-C dose $(28.7 \mathrm{~J} / \mathrm{mL})$. A. acidoterrestris was not detected after UV-C treatments, therefore that data was omitted from figures, and the highest inactivation rate for these experiments in PBS-fibre was of $4.58 \pm 0.09 \mathrm{Log}$.

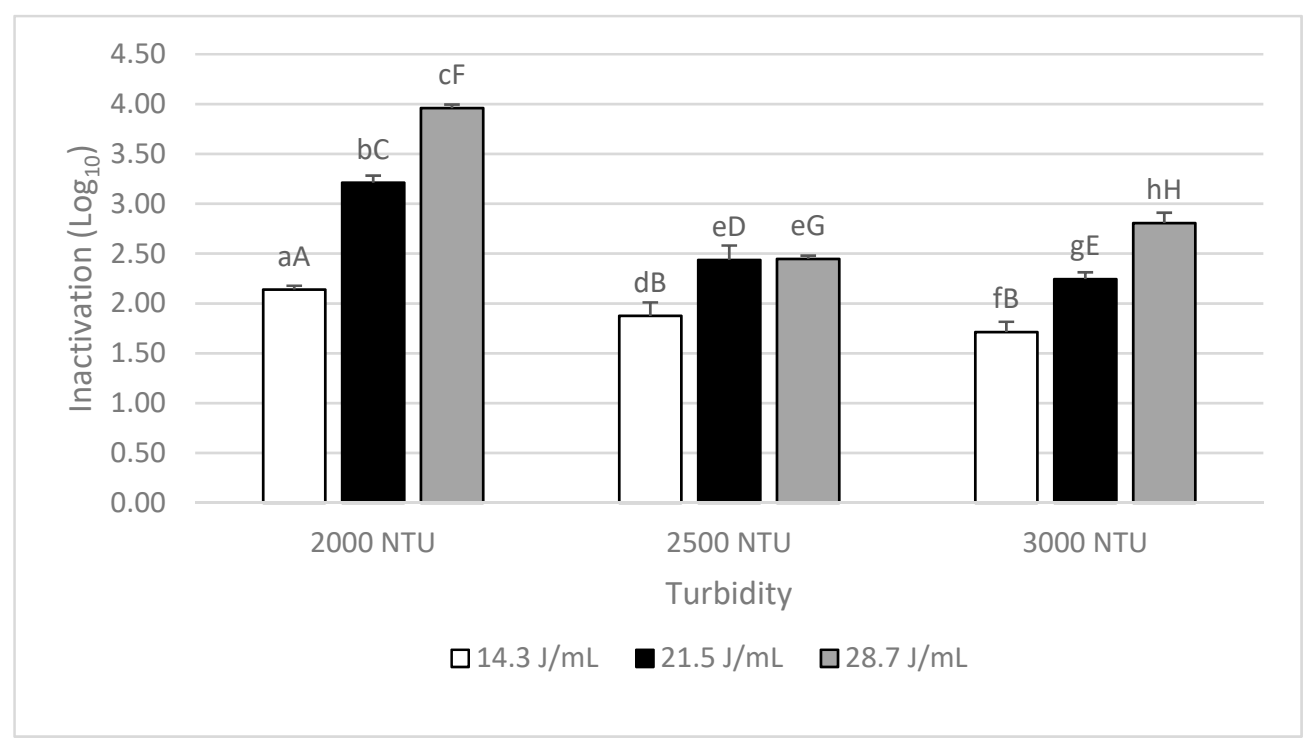

Figure 1. Inactivation of B. subtilis spores in PBS-fibre with different turbidity values modified with apple fibre. These suspensions were treated by UV-C at the doses of $14.3,21.5$, and $28.7 \mathrm{~J} / \mathrm{mL}$. Different lowercase letters represent significant differences $(p<0.05)$ between samples means of the same turbidity value but a different UV-C dose; capital letters represent significant differences between sample means of those ones treated with the same UV-C dose but with a different turbidity value.

\subsection{A. acidoterrestris Inactivation in Cloudy Apple Juice with Ascorbic Acid}

Cloudy apple juice with ascorbic acid presented high values of interferences coming from turbidity and $\alpha_{254}$. As an attempt to increase the probability of particles to get into a closer proximity to the UV-C source, the matrix was re-circulated setting the peristaltic pump at $500 \mathrm{rpm}$ (pumping speed of $0.708 \mathrm{~m} / \mathrm{s}$ ), that produces a flow rate of $64.4 \mathrm{~mL} / \mathrm{s}$ [15] which yields a dose of $0.42 \mathrm{~J} / \mathrm{mL}$ every pass. The number of passes and retention time for every given dose can be found in Table 1 . The result from these experiments can be seen in Figure 2, where the different doses were applied in a single pass and recirculating the sample until reaching the desired dose. Results of $A$. acidoterrestris lethality showed that this equipment is ineffective in a matrix with these interference characteristics if treated 
by a single pass and no significant differences were observed when UV-C dose increased. Nevertheless, different results were observed in treatments with recirculation where an inactivation of more than 5 Log was observed in the treatment at $125 \mathrm{~J} / \mathrm{mL}$, in which no surviving cells were detected on culture plates.

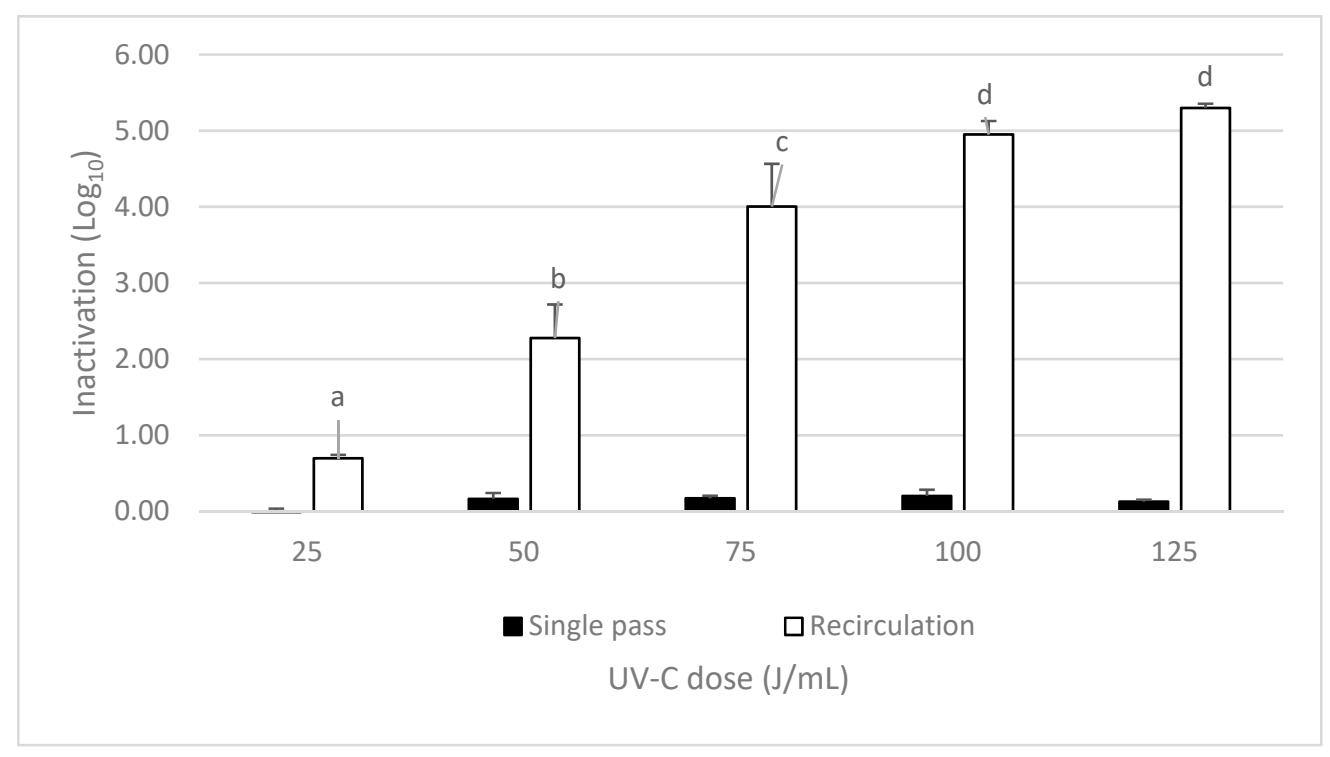

Figure 2. Inactivation of $A$. acidoterrestris inoculated in cloudy apple juice with ascorbic acid by UV-C at different doses $(25,50,75,100$, and $125 \mathrm{~J} / \mathrm{mL})$ in a single pass (black bars) or in recirculation (white bars). Error bars mark the standard deviation, and letters represent significant differences $(p<0.05)$ between sample means of the same treatment. No letters mean that there were no significant differences found between doses.

The data gathered from UV-C experiments with recirculation were utilized for adjusting to an inactivation model using GInaFiT software. Several inactivation models were assayed, from which it was selected the one with the highest $R^{2}(0.9772)$ and the lowest RMSE (0.3293), being that the Geeraerd shoulder tail model [21] (Figure 3), with an equation (Equation (2)). This was used to calculate the inactivation kinetics of $A$. acidoterrestris spores in this type of apple juice. The analysis of the inactivation kinetics allowed to obtain the $4 \mathrm{D}$ value of $76.25 \mathrm{~J} / \mathrm{mL}$, that was the estimated dose required to inactivate $4 \log$ of $A$. acidoterrestris spores in apple juice under these treatment conditions.

\subsection{Physicochemical Changes from the Application of UV-C Treatments with Recirculation}

Apple juice was irradiated with higher doses than those ones used to inactivate $A$. acidoterrestris spores in order to elucidate the changes that the continuous application of UV-C with recirculation can cause on some of the physicochemical properties of the apple juice with added ascorbic acid. The first observable change was an increase of browning of juice after being irradiated from $100 \mathrm{~J} / \mathrm{mL}$ onwards, as shown in Figure 4. There was also an augment of the turbidity value of the UV-C-treated samples and that became higher with higher doses. Contrary to that, $\alpha_{254}$ value of samples decreased with higher UV-C doses. 


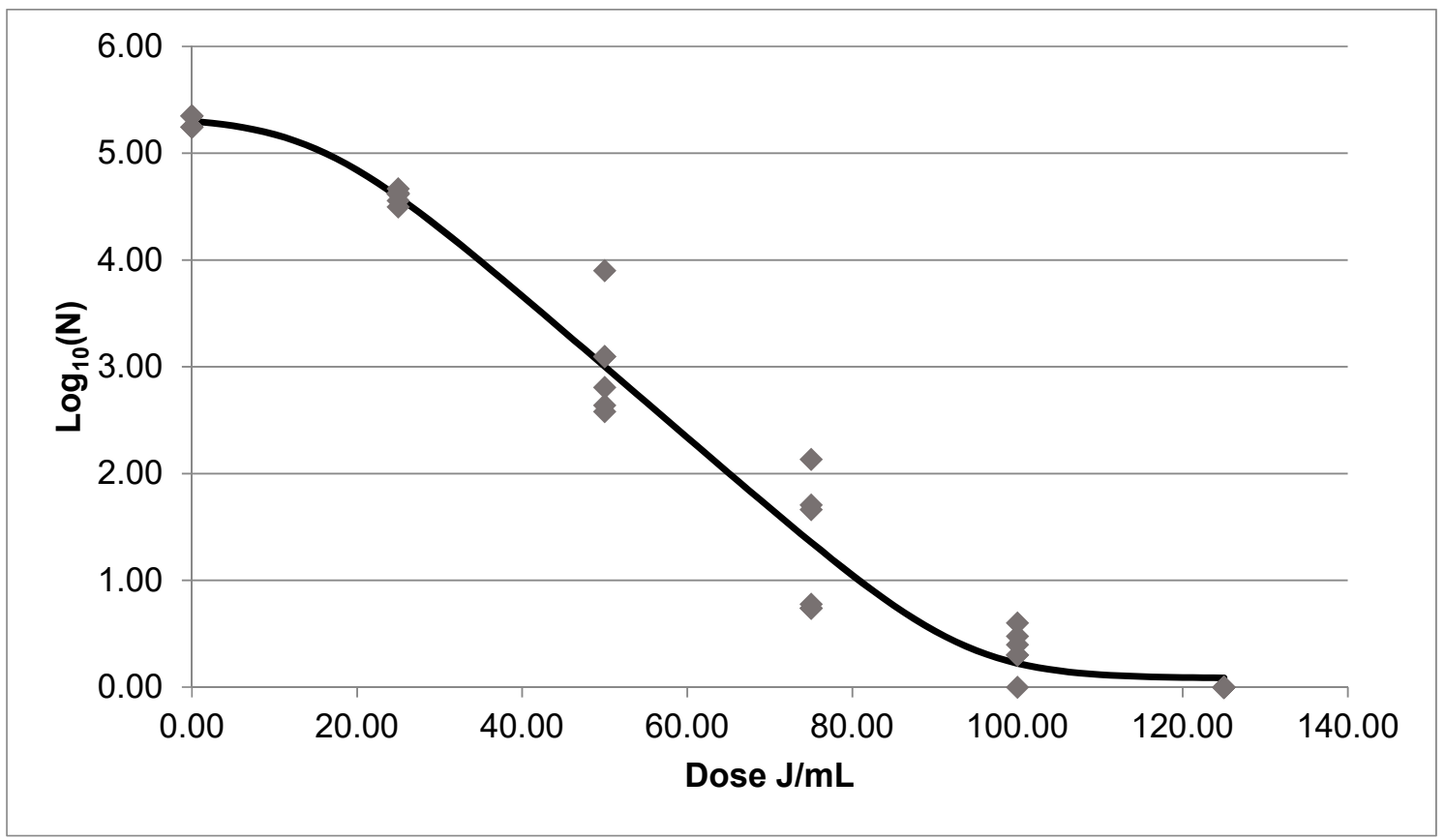

Figure 3. Inactivation kinetics of $A$. acidoterrestris in cloudy apple juice treated by UV-C with recirculation according to Geeraerd shoulder tail model obtained by using GInaFIT plug-in.

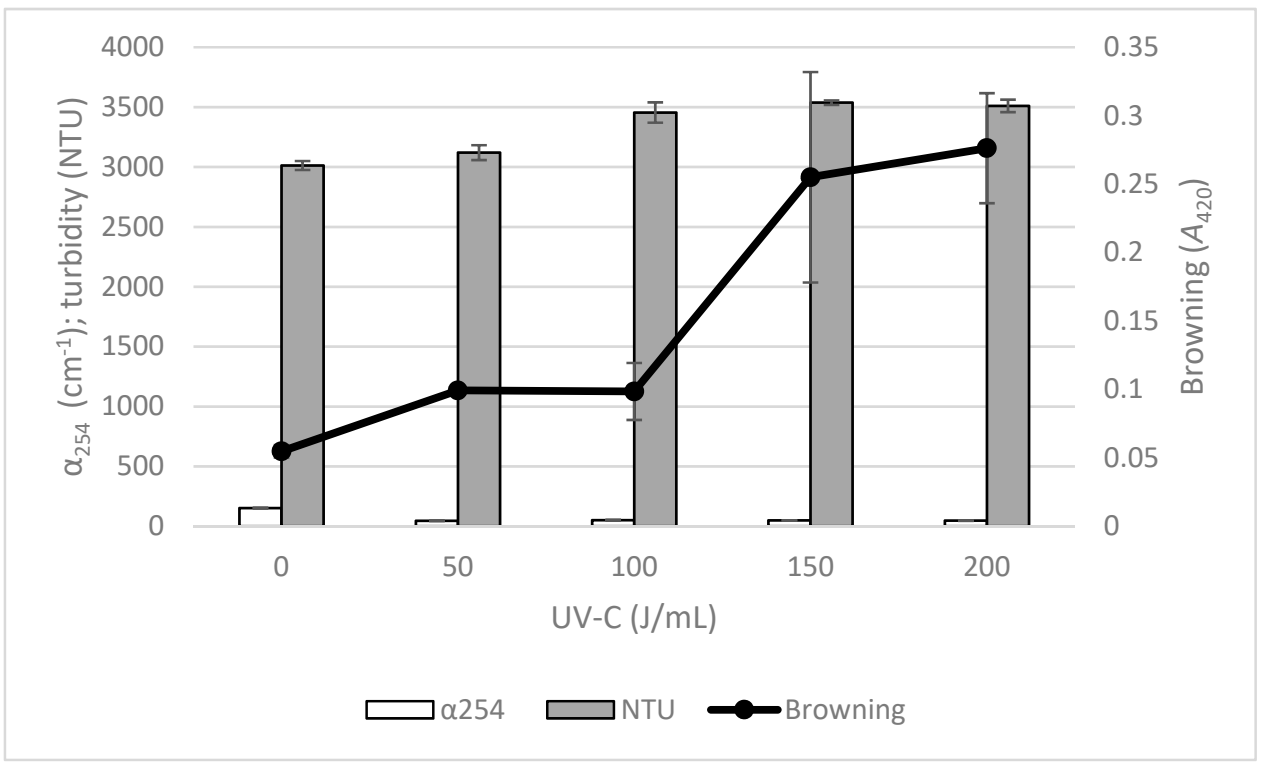

Figure 4. Physicochemical changes in apple juice with added ascorbic acid after UV-C treatments applied with recirculation at different doses $(50,100,150$, and $200 \mathrm{~J} / \mathrm{mL}) . \alpha_{254}\left(\mathrm{~cm}^{-1}\right)$ is represented by white bars, turbidity (NTU) is represented in grey bars, and browning (unitless) is represented by the plot line. Error bars mark the standard deviation between samples mean.

\section{Discussion}

B. subtilis spores became harder to inactivate as turbidity value increased in fibre suspensions (Figure 1). A similar behaviour was observed in a previous experiment [3] where caramel was added to PBS to modify its absorbance coefficient, showing that even slight $\alpha_{254}$ increments can drastically reduce the inactivation of $B$. subtilis spores by UV-C. As bacterial spores, vulnerability of fungal ascospores to UV-C is also higher when there are no interferences in the matrix, as it was also observed in a previous experiment with plain PBS [11]. In assays with PBS-fibre and A. acidoterrestris, spores could not be detected 
after most of UV-C treatments at any of the assayed turbidity values and comparing it with the results obtained from the same experiments with $B$. subtilis spores, it was found that $B$. subtilis spores were more UV-C-resistant than those of $A$. acidoterrestris. These results can also be contrasted with those of previous research [3] in which $B$. subtilis spores show a higher UV-C-tolerance compared with other bacterial spores including A. acidoterrestris in plain PBS.

Previous experiments with cloudy apple juice [4] measured its $\alpha_{254}$ at $11.54 \mathrm{~cm}^{-1}$, while in our experiments with the same type of apple juice but with added ascorbic acid set the value of $\alpha_{254}$ at $152.1 \mathrm{~cm}^{-1}$ (Figure 4). It is known that ascorbic acid has its absorption peak within the deep UV spectra between 243.5 and $265.5 \mathrm{~nm}$ [22], therefore it is reasonable to think that the addition of ascorbic acid, which is a common practice in fruit juice production, would represent an immediate interference against UV-C. The approach of a thin-film UV-C reactor has the intention of reducing the depth in which radiation has to travel through and to reach as many cells as possible to inactivate them, being most likely affected the closest ones to the UV-C source. These reactors work under laminar flow conditions, which means that liquids travel throughout the system in a layered way. If the properties of the matrix do not allow UV-C to reach deep into the matrix the microorganisms present in the farther layers of liquid from the UV-C radiation source will not be reached and affected. Similar results to those shown in Figure 2 were observed in a previous research [15] in which B. subtilis spores were treated by using the same recirculation approach on whole and skim milk reporting higher inactivation rates as the re-circulation and doses augmented surpassing 4-Log inactivation after a $100 \mathrm{~J} / \mathrm{mL}$ treatment, which is significantly higher than the results with the same dose in a single pass. That could have happened because recirculation allowed the sample to mix before re-entering the circuit, increasing the chances for the spores present in the matrix to get into a closer distance from the UV-C source and to be affected by it. The Reynolds number inside the UV-C reactor was considerably higher in samples treated with recirculation (Re $=290.8)$, but although that type of flow still corresponded to the range of laminar flow $(R e<2100)$ it might have propitiated a better exposure of the whole matrix as the fluid layers closer to the UV-C source were different at each pass. Another factor that could have contributed to a better inactivation of $A$. acidoterrestris spores could be that higher UV-C doses decreased the $\alpha_{254}$ value of the samples (Figure 4) which also might have decreased the matrix absorption of UV light. Comparing results between the experiments with PBS-fibre and juice, it could be presumed that the most limiting factor of the matrix in a UV-C treatment could be the $\alpha_{254}$ value, even more than the interference that turbidity could represent. The "Geeraerd model with shoulder and tail" inactivation model obtained for A. acidoterrestris in this type of apple juice [21] (Figure 4) showed that there was a slow spore inactivation at the beginning of the treatment followed up by a later exponential increase. Similar experiments on B. subtilis spores inoculated into milk and treated by UV-C [15] working under the same settings showed a better adjustment to a "Weibull with tail" inactivation model [23]. A tail in the model represents a slow-down or a less exponential inactivation of spores after reaching certain dose in a treatment.

The changes observed in UV-C-treated apple juice on its $\alpha_{254}$ value could have been the product of a prolonged exposure to UV-C that can degrade ascorbic acid into dehydroascorbic acid and to 2,3-diketogulonic acid [24,25], compounds that have different absorbance peaks [26]. The addition of ascorbic acid was the main responsible for the increase of $\alpha_{254}$ in apple juice, and also for the inhibition of the polyphenol oxidase (PPO) activity at the moment of the juice extraction. Therefore, when lower concentrations of ascorbic acid were available as a consequence of photodegradation, it might have not been able to inhibit the activity of that enzyme [27]. Although some investigation show that UV radiation can also inhibit PPO, those treatments have not been able to achieve a total inactivation of the enzyme [28]. In our experiments a possible degradation of the ascorbic acid in apple juice, as well as a higher contact with oxygen caused by recirculation, might have enabled PPO to oxidise phenolic compounds into the pigmented ones (o-quinones) [9]. 
Turbidity might have been affected as well by the degradation of ascorbic acid due to the oxidation of polyphenols that would lead to polymerise and react with proteins, and consequently increasing the formation of larger size particles [29].

\section{Conclusions}

The presence of spoiling bacteria in juices, not only in their vegetative state, but also in the state of spores, needs to be addressed by the emerging technologies intended for these products' pasteurisation or sterilization, especially resistant spores, like those of $A$. acidoterrestris, that could cause big losses of product and money, deserve special attention. Fruit matrices themselves can oppose different types and levels of interference depending on their singular properties and it is important to understand at what degree those interferences can affect the efficiency of UV-C treatments. The equipment features also have a great influence on the performance of the treatments. Cloudy apple juice presents a high turbidity value, but addition of ascorbic acid at the moment of extraction, which is a common practice in fruit juice industry, also increases absorption coefficient at $254 \mathrm{~nm}$ what should not be ignored when research about the effects of UV-C treatments in this type of product is performed. The results from the experiments hereby suggest that, although the degree of turbidity can affect the effectiveness of UV-C in inactivating resistant spores, that type of interference might not be as determinant as it is the absorption coefficient. There are other factors to take also into consideration that could affect the quality of the final product like potential browning reactions. The findings derived from this research could help in the development of new designs of UV-C reactors suitable to treat a wider spectrum of fruit juices as well as other liquid foods, and also explore their potential combination with other technologies that could increase the inactivation, not only inactivating microorganisms, but also enzymes like PPO, which in turn could reduce the intensity at which UV-C treatments would need to be applied to foods.

Author Contributions: Conceived and designed the experiments: J.N.S.-G., M.M.-G., M.M.H.-H., R.G., and A.X.R.-S.; performed the experiments: J.N.S.-G.; analysed the data: J.N.S.-G., R.G., and A.X.R.-S.; wrote the paper: J.N.S.-G., R.G., and A.X.R.-S. All authors have read and agreed to the published version of the manuscript.

Funding: This research was funded by the Spanish Ministry of Economy and Competitiveness, project AGL2014-60005-R.

Institutional Review Board Statement: Not applicable.

Informed Consent Statement: Not applicable.

Data Availability Statement: Not applicable.

Acknowledgments: The authors acknowledge the financial support received from the research project AGL2014-60005-R of the Spanish Ministry of Economy and Competitiveness, along with the grant number 440,526 given to Jezer Noé Sauceda Gálvez by CONACyT (Consejo Nacional de Ciencia y Tecnología, México) and from INAPI (Instituto de Apoyo a la Investigación e Innovación) of Sinaloa.

Conflicts of Interest: The authors declare no conflict of interest.

\section{References}

1. De Souza, P.M.; Fernández, A. Effects of UV-C on physicochemical quality attributes and Salmonella enteritidis inactivation in liquid egg products. Food Control 2011, 22, 1385-1392. [CrossRef]

2. Martín, M.F.S.; Barbosa-Cánovas, G.V.; Swanson, B.G. Food Processing by High Hydrostatic Pressure. Crit. Rev. Food Sci. Nutr. 2002, 42, 627-645. [CrossRef]

3. Reverter-Carrión, L.; Sauceda-Gálvez, J.; Codina-Torrella, I.; Hernández-Herrero, M.; Gervilla, R.; Roig-Sagués, A. Inactivation study of Bacillus subtilis, Geobacillus stearothermophilus, Alicyclobacillus acidoterrestris and Aspergillus niger spores under Ultra-High Pressure Homogenization, UV-C light and their combination. Innov. Food Sci. Emerg. Technol. 2018, 48, 258-264. [CrossRef] 
4. Sauceda-Gálvez, J.; Tió-Coma, M.; Martinez-Garcia, M.; Hernández-Herrero, M.; Gervilla, R.; Roig-Sagués, A. Effect of single and combined UV-C and ultra-high pressure homogenisation treatments on inactivation of Alicyclobacillus acidoterrestris spores in apple juice. Innov. Food Sci. Emerg. Technol. 2020, 60, 102299. [CrossRef]

5. Bevilacqua, A.; Cibelli, F.; Corbo, M.R.; Sinigaglia, M. Effects of high-pressure homogenization on the survival of Alicyclobacillus acidoterrestris in a laboratory medium. Lett. Appl. Microbiol. 2007, 45, 382-386. [CrossRef]

6. Lee, S.-Y.; Dougherty, R.H.; Kang, D.-H. Inhibitory Effects of High Pressure and Heat on Alicyclobacillus acidoterrestris Spores in Apple Juice. Appl. Environ. Microbiol. 2002, 68, 4158-4161. [CrossRef] [PubMed]

7. Chang, S.-S.; Kang, D.-H. Alicyclobacillusspp. in the Fruit Juice Industry: History, Characteristics, and Current Isolation/Detection Procedures. Crit. Rev. Microbiol. 2004, 30, 55-74. [CrossRef] [PubMed]

8. Walker, M.; Phillips, C. Alicyclobacillus acidoterrestris: An increasing threat to the fruit juice industry? Int. J. Food Sci. Technol. 2007, 43, 250-260. [CrossRef]

9. Koutchma, T.; Popović, V.; Ros-Polski, V.; Popielarz, A. Effects of Ultraviolet Light and High-Pressure Processing on Quality and Health-Related Constituents of Fresh Juice Products. Compr. Rev. Food Sci. Food Saf. 2016, 15, 844-867. [CrossRef]

10. Choudhary, R.; Bandla, S. Ultraviolet Pasteurization for Food Industry. Int. J. Food Sci. Nutr. Eng. 2012, 2, 12-15. [CrossRef]

11. Sauceda-Gálvez, J.; Roca-Couso, R.; Martinez-Garcia, M.; Hernández-Herrero, M.; Gervilla, R.; Roig-Sagués, A. Inactivation of ascospores of Talaromyces macrosporus and Neosartorya spinosa by UV-C, UHPH and their combination in clarified apple juice. Food Control. 2019, 98, 120-125. [CrossRef]

12. Adzahan, N.M.; Lau, P.L.; Hashim, N.; Shamsudin, R.; Sew, C.C.; Sobhi, B. Pineapple juice production using ultraviolet pasteurisation: Potential cost implications. J. Agribus. Mark. 2011, 4, 38-50.

13. Koutchma, T.; Keller, S.; Chirtel, S.; Parisi, B. Ultraviolet disinfection of juice products in laminar and turbulent flow reactors. Innov. Food Sci. Emerg. Technol. 2004, 5, 179-189. [CrossRef]

14. Harwood, C.R. Bacillus Subtilis as a Model for Bacterial Systems Biology. In Encyclopedia of Life Sciences; John Wiley \& Sons, Ltd.: Chichester, UK, 2007.

15. Martinez-Garcia, M.; Sauceda-Gálvez, J.N.; Codina-Torrella, I.; Hernández-Herrero, M.M.; Gervilla, R.; Roig-Sagués, A.X. Evaluation of Continuous UVC Treatments and its Combination with UHPH on Spores of Bacillus subtilis in Whole and Skim Milk. Foods 2019, 8, 539. [CrossRef] [PubMed]

16. Gervilla, R.; Salas, F.; Salas, J.; Guamis, A.; Guamis, D.; Moreta, L. System and Method for Sterilizing A Fluid. European Patent Application No. EP-2965766-A1, 11 November 2014.

17. Rahn, R.O. Potassium Iodide as a Chemical Actinometer for $254 \mathrm{~nm}$ Radiation: Use of Iodate as an Electron Scavenger. Photochem. Photobiol. 1997, 66, 885. [CrossRef]

18. Ozbayoglu, M.E.; Omurlu, C. Analysis of the effect of eccentricity on the flow characteristics of annular flow of non-Newtonian fluids using finite-element method. In Proceedings of the SPE/ICoTA Coiled Tubing and Well Intervention Conference and Exhibition, The Woodlands, TX, USA, 4-5 April 2006; Volume 2006, pp. 293-298.

19. Chang, S.; Kang, D.-H. Development of novel Alicyclobacillus spp. isolation medium. J. Appl. Microbiol. 2005, 99, 1051-1060. [CrossRef] [PubMed]

20. Meydav, S.; Saguy, I.; Kopelman, I.J. Browning determination in citrus products. J. Agric. Food Chem. 1977, 25, 602-604. [CrossRef]

21. Geeraerd, A.; Herremans, C.; Van Impe, J. Structural model requirements to describe microbial inactivation during a mild heat treatment. Int. J. Food Microbiol. 2000, 59, 185-209. [CrossRef]

22. Berg, R.W. Investigation of L(+)—Ascorbic acid with Raman spectroscopy in visible and UV light. Appl. Spectrosc. Rev. 2014, 50, 193-239. [CrossRef]

23. Albert, I.; Mafart, P. A modified Weibull model for bacterial inactivation. Int. J. Food Microbiol. 2005, 100, 197-211. [CrossRef]

24. Falguera, V.; Garvín, A.; Garza, S.; Pagán, J.; Ibarz, A. Effect of UV-Vis Photochemical Processing on Pear Juices from Six Different Varieties. Food Bioprocess Technol. 2014, 7, 84-92. [CrossRef]

25. Tikekar, R.V.; Anantheswaran, R.C.; Elias, R.J.; Laborde, L.F. Ultraviolet-Induced Oxidation of Ascorbic Acid in a Model Juice System: Identification of Degradation Products. J. Agric. Food Chem. 2011, 59, 8244-8248. [CrossRef] [PubMed]

26. Kleinwächter, M.; Selmar, D. A novel approach for reliable activity determination of ascorbic acid depending myrosinases. $J$. Biochem. Biophys. Methods 2004, 59, 253-265. [CrossRef] [PubMed]

27. Almeida, M.E.M.; Nogueira, J.N. The control of polyphenol oxidase activity in fruits and vegetables. Plant Foods Hum. Nutr. 1995, 47, 245-256. [CrossRef] [PubMed]

28. Guerrero-Beltrán, J.A.; Barbosa-Cénovas, G.V. Inactivation of Saccharomyces cerevisiae and polyphenoloxidase in mango nectar treated with UV light. J. Food Prot. 2006, 69, 362-368. [CrossRef]

29. Kolniak-Ostek, J.; Oszmiański, J.; Wojdyło, A. Effect of 1-ascorbic acid addition on quality, polyphenolic compounds and antioxidant capacity of cloudy apple juices. Eur. Food Res. Technol. 2013, 236, 777-798. [CrossRef] 